\title{
Kaempferol Protects Mice from D-GalN/LPS- induced Acute Liver Failure by Regulating the Autophagy Pathway
}

Yuan Tian

Capital Medical University Youan Hospital

Feng Ren

Capital Medical University Youan Hospital

Ling Xu

Capital Medical University Youan Hospital

Weihua Li

Capital Medical University Youan Hospital

Mei Liu

Capital Medical University Youan Hospital

Jianhua Hu

Capital Medical University Youan Hospital

Xiangying Zhang ( $\nabla$ zhangxy2018@ccmu.edu.cn )

beijing youan hospital

\section{Research}

Keywords: Kaempferol, acute liver failure, autophagy, liver inflammation, D-

galactosamine/lipopolysaccharide

Posted Date: December 31st, 2020

DOl: https://doi.org/10.21203/rs.3.rs-137375/v1

License: (a) (1) This work is licensed under a Creative Commons Attribution 4.0 International License.

Read Full License 


\section{Abstract \\ Background}

Kaempferol, a flavonoid compound present in many edible plants, has been used in traditional medicine and has various biological functions. Acute liver failure (ALF) is a lethal clinical syndrome with severe liver function damage. There are currently no effective treatments for ALF except for liver transplantation. The aim of this study is to explored the mechanisms underlying the therapeutic effect of kaempferol in ALF.

\section{Methods}

The ALF mouse model was established using D-galactosamine (D-GalN, $700 \mathrm{mg} / \mathrm{kg}$ )/lipopolysaccharide (LPS, $10 \mu \mathrm{g} / \mathrm{kg}$ ). Two hours before the administration of D-GalN/LPS, different group of mice were pretreated according different doses of kaempferol, 6 hours after injection of D-GaIN/LPS, and then killed. The survival rate, liver function and inflammatory cytokine levels were assessed. It was determined whether kaempferol pretreatment protected hepatocytes from ALF induced by D-GaIN/LPS via autophagy pathway in vivo and in vitro.

\section{Results}

Pretreatment with a high dose of kaempferol significantly decreased the survival rate and increased severe liver damage; however, pretreatment with a low dose of kaempferol showed the opposite effect. Furthermore, pretreatment with a high dose of kaempferol augment the levels of proinflammatory cytokines and markers of the MAPK signaling pathway, while pretreatment with a low dose of kaempferol showed the opposite effect. In addition, pretreatment with a high dose of kaempferol decreased autophagy, but pretreatment with a low dose of kaempferol increased autophagy in vivo and in vitro. It was also proved that pretreatment with 3-methyadenine (3-MA) or Atg7 siRNA to inhibit autophagy partially negated the hepatoprotective effect of kaempferol $(5 \mathrm{mg} / \mathrm{kg})$ pretreatment in ALF mice induced by D-GaIN/LPS.

\section{Conclusions}

Our findings demonstrate that effects of different doses of kaempferol on D-GaIN/LPS-induced ALF is remarkably different by regulating the autophagy pathway. Therefore, we should consider selecting the optimal dose of kaempferol as a potential treatment method for patients with ALF.

\section{Background}


Acute liver failure (ALF) is a fatal hepatic disease associated with rapid loss of liver function resulting in multiorgan dysfunction, encephalopathy and coagulopathy in patients. This critical illness causes high mortality and morbidity. When it occurs, the survival of patients only depends on emergency liver transplantation [1]. Therefore, an effective drug to treat acute liver failure is urgently needed.

Kaempferol is a flavonoid that mainly extracted from the root of Kaempferia galanga L., it is widely present in various natural plants, fruits, vegetables, beverages and teas [2, 3]. Kaempferol possesses several pharmacological activities, including cardioprotective, neuroprotective, antioxidative, antidiabetic and anticarcinogenic properties [4]. A growing number of studies suggest that kaempferol can reduce the risk of developing various cardiovascular diseases, diabetes, cancer, and so on [5-7]. Studies have found that kaempferol is related to the treatment of many diseases. For instance, in a time- and concentrationdependent manner, kaempferol can decrease HeLa cervical cancer cell viability and induce apoptosis by remarkably restrain the PI3K/AKT pathway [8]. Studies also demonstrated that kaempferol distinctly inhibited bladder cancer EJ cells growth by inducing $S$ cell cycle arrest and apoptosis and increased expression level of phosphorylated status of p53 by regulating mitochondria-mediated apoptotic signaling pathways [9].

Kaempferol are traditionally recognized as potential protective effects on liver injury [10-12] and was shown to be effective for anti-inflammatory properties in liver cells [13]. In addition, kaempferol has been demonstrated to significantly alleviate acute liver injury (ALI), inflammation and early hepatocyte apoptosis caused by propacetamol [14]. Our previous study showed that kaempferol pretreatment could alleviate liver damage in D-galactosamine (D-GalN)/lipopolysaccharide (LPS) -induced mice [15]. However, the underlying therapeutic mechanisms of kaempferol in ALF are still unclear.

Autophagy is an intracellular catabolic pathway with highly conserved where biomolecules and organelles can be degraded by the lysosomes. Autophagy is crucial for maintaining homeostasis of cell and replenish many types of substances for cell survival under stressful conditions [16]. Autophagy is closely associated with liver disease. Previous reports have revealed that autophagy may suppress the growth of tumors in chronic liver disease, and impaired autophagy can lead to a significant increase of glycolysis in liver cancer [17]. In addition, autophagy can protect against the accumulation of fat in hepatocytes during nonalcoholic fatty liver disease (NAFLD) [18]. In our recent study, we explored that autophagy activation protected mice from ALF by inhibiting glycogen synthase kinase $3 \beta$ (GSK3 $\beta$ ) activity [19]. In current therapeutic research, the problem of drug toxicity has always existed. There are few studies on the effects of different doses of kaempferol on ALF. Therefore, the toxicity of kaempferol requires further study.

Given the above information, in this study, A model of ALF induced by D-GaIN/LPS- mouse is used and has been widely used to explore the underlying mechanisms involved in potential therapeutic drugs for clinical use in treating ALF [20,21], and the functional effects of different doses of kaempferol and related regulatory pathways were evaluated in the context of ALF. Our findings demonstrated that a dose 
of kaempferol resulted in severe liver injury, whereas a low dose of kaempferol protected against ALF induced by D-GalN/LPS via regulating the autophagy pathway.

\section{Materials And Methods}

\section{Animals and treatments}

Male wild-type mice (C57BL/6, 8-12 weeks old) were purchased from Capital Medical University (CMU), keep them in the $\mathrm{CMU}$ animal facility under conditions free of specific pathogens, and subjected to humane care guidelines as required by the CMU Animal Care Committee. The animal experiment protocol was approved by the Institutional Animal Care and Use Committee (IACUC) of CMU. The mice were injected intraperitoneally with D-GalN (700 mg/kg; Sigma, St. Louis, MO, USA) and LPS (10 $\mu \mathrm{g} / \mathrm{kg}$; InvivoGen, San Diego, CA, USA) to induce ALF, or were intraperitoneally or intravenously injected with the same volume of normal saline volume of saline in the all experimental group. Two hours before the administration of D-GalN/LPS, mice were pretreated with different doses $(2.5 \mathrm{mg} / \mathrm{kg}, 5 \mathrm{mg} / \mathrm{kg}, 10 \mathrm{mg} / \mathrm{kg}$, $20 \mathrm{mg} / \mathrm{kg}$ and $40 \mathrm{mg} / \mathrm{kg}$ ) of kaempferol (Sigma, St. Louis, Missouri, USA) via tail vein injection. 3Methyladenine (3-MA) can be used to block autophagy, while chloroquine (CQ) is a commonly used chemical drug that inhibits the fusion of lysosomes and autophagosomes, which contributes to observe the autophagic flux. Consequently, 3-MA (10 mg/kg; Sigma, St. Louis, MO, USA) and CQ $(60 \mathrm{mg} / \mathrm{kg}$; Sigma, St. Louis, MO, USA) were administered intraperitoneally to mice $2 \mathrm{~h}$ before the administration of $\mathrm{D}$ GaIN/LPS. The mice were anesthetized, and liver and blood samples were collected for further analysis.

\section{Serum aminotransferase activity}

Blood samples were collected from the mice at $6 \mathrm{~h}$ after D-GalN/LPS administration. Serum levels of alanine aminotransferase (ALT) and aspartate aminotransferase (AST), which are markers of hepatic damage, were measured using a multiparametric analyzer (AU 5400, Olympus, Japan) according to the manufacturer's protocol.

\section{Histopathological analysis}

According to a standard protocol, liver samples were fixed in formalin and embedded in paraffin wax, and sections were stained using hematoxylin and eosin (H\&E) for histopathological evaluation, which carried out under light microscopy.

\section{Quantitative reverse-transcription polymerase chain reaction (qRT-PCR)}

Total RNA was isolated from liver tissue using TRIzol reagent following the protocol of manufacturer. Reverse transcription of RNA $(2.5 \mu \mathrm{g})$ into cDNA using the SuperScript III first-strand synthesis system (Invitrogen, Carlsbad, CA, USA). Use DNA Engine with a Chromo 4 detector (MJ Research, Waltham, MA) for qRT-PCR. The final reaction volume was $20 \mu$, including $1 \times$ super mix (Platinum SYBR Green qPCR kit, Invitrogen, Carlsbad, CA), $2 \mu \mathrm{l}$ of cDNA and $0.5 \mu \mathrm{l}$ of each primer. The amplification conditions were as 
follows: $50^{\circ} \mathrm{C}(2 \mathrm{~min}), 95^{\circ} \mathrm{C}(5 \mathrm{~min})$, and 50 cycles of $95^{\circ} \mathrm{C}(15 \mathrm{~s})$ and $60^{\circ} \mathrm{C}(30 \mathrm{~s})$. The mRNA levels were calculated using the $2^{-\Delta \Delta \mathrm{Ct}}$ method.

\section{Western blotting}

Liver tissue samples or cells were lysed in radioimmunoprecipitation assay (RIPA) buffer containing phosphatase and protease inhibitors. A total of $20 \mu \mathrm{g}$ of protein in each sample was separated by $12 \%$ sodium dodecyl sulfate-polyacrylamide gel electrophoresis at $80 \mathrm{~V}$ for 30 minutes and $120 \mathrm{~V}$ for $1 \mathrm{~h}$, and then use the Bio-Rad blotting transfer system to electrotransfer to PVDF membrane (Bio-Rad, Hercules, CA). Monoclonal rabbit antibodies against $\beta$-actin, p-JNK, p-ERK, p-p38, LC3B, ATG7, and p62 were appropriately diluted $(1: 1,000)$ in $10 \mathrm{ml}$ of blocking buffer at $4{ }^{\circ} \mathrm{C}$ overnight. After washing the membranes with Tris-buffered saline with Tween-20 (TBST), the appropriate horseradish peroxidaseconjugated secondary antibody (1:2000) in $10 \mathrm{ml}$ of blocking buffer was added and incubated for $1 \mathrm{~h}$ at room temperature. Then the membranes were washed 3 times with TBST for $30 \mathrm{~min}$. Next, An enhanced chemiluminescence commercial kit (Thermo Fisher Scientific, Inc., Rockford, IL, USA) was used for detection of the proteins. Image J software (National Institutes of Health, New York, NY, USA) was used for quantification of the western blotting results.

\section{Atg7 small interfering RNA treatment in vivo}

siRNA (3 mg/kg; Jima, Suzhou) and an Entranster ${ }^{\text {min }}$ in vivo transfection reagent (Engreen Biosystem Co, Beijing) were used to knock out Atg7 by hydrodynamic tail vein injection in mice. Scrambled siRNA ( $3 \mathrm{mg} / \mathrm{kg}$ ) was used as a control. These steps were performed in accordance with the procedures of manufacturer.

\section{Isolation of primary mouse hepatocytes}

Hanks' solution containing collagenase was used to perfusion of mouse livers when the mice were 7 weeks old, and as mentioned earlier, live hepatocytes are separated by Percoll isocratic centrifugation [22].

\section{Starvation-induced autophagy in vitro.}

The most robust condition for inducing autophagy is starvation. Primary hepatocytes were transfected with the GFP-LC3 plasmid for $12 \mathrm{~h}$, and then incubated in Earle's balanced salt solution for different times to starve their amino acids. The percentage of cells were calculate with GFP-LC3 puncta in the different treatment groups. GFP-positive cells were regared as cells that showed bright, punctate staining. About 50 cells were counted, and the experiment was repeated at least three times.

\section{Statistical analysis}

Results from three independent experiments were presented as the mean \pm SD. Differences between two groups were analyzed using unpaired Student's t-tests by using GraphPad Prism 7 Software. P of values $<0.05$ were considered statistically significant. 


\section{Results}

\section{Effects of kaempferol on D-GalN/LPS-induced ALF}

First, we examined the effects of different doses of kaempferol on ALF induced by D-GalN/LPS in mice. Different doses of kaempferol ( $2.5 \mathrm{mg} / \mathrm{kg}, 5 \mathrm{mg} / \mathrm{kg}, 10 \mathrm{mg} / \mathrm{kg}, 20 \mathrm{mg} / \mathrm{kg}$ and $40 \mathrm{mg} / \mathrm{kg})$ were intraperitoneally administrated. As shown in the survival analysis (Fig. 1a), at $5 \mathrm{~h}$ after D-GalN/LPS injection, mice in the $20 \mathrm{mg} / \mathrm{kg}$ kaempferol group began to die, their survival rate was $0 \%$ ( 0 of 20 mice). In addition, the survival rates of the $2.5 \mathrm{mg} / \mathrm{kg}$ kaempferol, $10 \mathrm{mg} / \mathrm{kg}$ kaempferol and D-GaIN/LPS groups were $25 \%$ (5 of 20 mice), $30 \%$ ( 6 of 20 mice) and $20 \%$ (4 of 20 mice), respectively, at $24 \mathrm{~h}$. In contrast, the survival rate of the $5 \mathrm{mg} / \mathrm{kg}$ kaempferol group stabilized at $80 \%$ (16 of 20 mice) at $24 \mathrm{~h}$.

Pretreatment with $20 \mathrm{mg} / \mathrm{kg}$ and $40 \mathrm{mg} / \mathrm{kg}$ kaempferol exhibited higher serum ALT (sALT) and serum AST (sAST) levels than group of D-GalN/LPS, but the rest of groups exhibited lower levels of SALT and SAST, and especially the $5 \mathrm{mg} / \mathrm{kg}$ kaempferol group exhibited the lowest levels (Fig. 1c). Consistent with the ALT and AST activities, the liver histopathology in the $20 \mathrm{mg} / \mathrm{kg}$ kaempferol group showed increased hepatocyte injury, similar to that of the D-GalN/LPS group, but mice in the groups of $2.5 \mathrm{mg} / \mathrm{kg}$ and $5 \mathrm{mg} / \mathrm{kg}$ kaempferol showed reduced hepatocyte injury, and the $5 \mathrm{mg} / \mathrm{kg}$ kaempferol group exhibited the lowest amount of injury (Fig. 1b). Moreover, we also explored the protective effects of kaempferol $(5 \mathrm{mg} / \mathrm{kg}$ ) pretreatment on liver injury in mice at $2 \mathrm{~h}, 4 \mathrm{~h}, 6 \mathrm{~h}$ and $8 \mathrm{~h}$ after D-GalN/LPS administration. The results showed that D-GaIN/LPS-induced serum ALT and AST levels significantly decreased by $5 \mathrm{mg} / \mathrm{kg}$ kaempferol at $4 \mathrm{~h}, 6 \mathrm{~h}$ and $8 \mathrm{~h}$ (Fig. 1d). These results demonstrate that high doses of kaempferol can induce more severe injury, while pretreatment with low doses of kaempferol significantly increase the survival rate and protect against ALF induced by D-GaIN/LPS.

\section{Effects of kaempferol on liver inflammation in mice with ALF induced by D-GalN/LPS}

Because our previous study suggested that the liver inflammatory response plays an important role in ALF [23], we sought to explore whether different effects on liver inflammation were induced by kaempferol at different doses in D-GaIN/LPS-induced ALF mice. As shown in Fig. 2a, compared with the D-GaIN/LPS group, the mice in pretreatment groups with $2.5 \mathrm{mg} / \mathrm{kg}$ and $5 \mathrm{mg} / \mathrm{kg}$ kaempferol showed lower gene expression levels of TNF-a, interleukin (IL)-6, IL-1ß, IL-12p40, C-X-C motif chemokine ligand (CXCL)-10 and CXCL-2 in the liver, and the $5 \mathrm{mg} / \mathrm{kg}$ kaempferol group exhibited the lowest levels. In contrast, pretreatment with $10 \mathrm{mg} / \mathrm{kg}$ and $20 \mathrm{mg} / \mathrm{kg}$ kaempferol resulted in notably increased genes expression levels of these cytokines. Additionally, the pretreatment groups of $2.5 \mathrm{mg} / \mathrm{kg}, 5 \mathrm{mg} / \mathrm{kg}$ and $10 \mathrm{mg} / \mathrm{kg}$ kaempferol showed higher gene expression levels of IL-10 than the group of D-GalN/LPS, among which the $5 \mathrm{mg} / \mathrm{kg}$ kaempferol group exhibited the highest expression. Pretreatment with kaempferol at $10 \mathrm{mg} / \mathrm{kg}$ and $20 \mathrm{mg} / \mathrm{kg}$ decreased the genes expression level of IL-10. Furthermore, we further confirmed whether the MAPK signaling pathway was affected by different doses of kaempferol. The protein expression levels of p-JNK, p-ERK and p-p38 were determined using Western blotting, and the 
groups of $2.5 \mathrm{mg} / \mathrm{kg}, 5 \mathrm{mg} / \mathrm{kg}$ and $10 \mathrm{mg} / \mathrm{kg}$ kaempferol exhibited reduced levels of these proteins, with the $5 \mathrm{mg} / \mathrm{kg}$ kaempferol group exhibiting the lowest levels. In contrast, pretreatment with $10 \mathrm{mg} / \mathrm{kg}$ and $20 \mathrm{mg} / \mathrm{kg}$ kaempferol resulted in increased levels (Fig. 2b). Therefore, these results confirmed that pretreatment with high doses of kaempferol can increase the hepatic inflammatory response and that pretreatment with low dose of kaempferol can significantly decrease the hepatic inflammatory response in ALF induced by D-GaIN/LPS.

\section{Effects of kaempferol on liver autophagy in mice with ALF induced by D-GalN/LPS}

Given that our previous studies suggested that autophagy plays a significant role in ALF induced by DGaIN/LPS [19], we explored that whether different doses of kaempferol affected liver autophagy in DGalN/LPS-induced ALF. Our data of qRT-PCR showed that (Fig. 3a), pretreatment with $2.5 \mathrm{mg} / \mathrm{kg}$, $5 \mathrm{mg} / \mathrm{kg}, 10 \mathrm{mg} / \mathrm{kg}$ and $20 \mathrm{mg} / \mathrm{kg}$ kaempferol significantly promoted the gene expression of LC3 compared with D-GalN/LPS-induced ALF in mice. Moreover, pretreatment with $2.5 \mathrm{mg} / \mathrm{kg}, 5 \mathrm{mg} / \mathrm{kg}$, and $20 \mathrm{mg} / \mathrm{kg}$ kaempferol also promoted the gene expression of Atg7, and the $5 \mathrm{mg} / \mathrm{kg}$ kaempferol group exhibited the highest expression. These data were consistent with western blot analyses. Pretreatment with $2.5 \mathrm{mg} / \mathrm{kg}, 5 \mathrm{mg} / \mathrm{kg}, 10 \mathrm{mg} / \mathrm{kg}$ and $20 \mathrm{mg} / \mathrm{kg}$ kaempferol resulted in increased protein expression of LC3, and the $5 \mathrm{mg} / \mathrm{kg}$ kaempferol group exhibited the highest expression. In addition, pretreatment with $2.5 \mathrm{mg} / \mathrm{kg}$ and $5 \mathrm{mg} / \mathrm{kg}$ kaempferol dramatically decreased the protein expression of $\mathrm{p} 62$, and $10 \mathrm{mg} / \mathrm{kg}$ and $20 \mathrm{mg} / \mathrm{kg}$ kaempferol significantly increased the protein expression of p62 (Fig. 3b). To further demonstrate that the effect of kaempferol $(5 \mathrm{mg} / \mathrm{kg})$ on autophagy flux, the fuse of lysosomes and autophagosomes was inhibited by CQ pretreatment. The CQ pretreatment did not further increased LC3II conversion and did not further degraded p62 compared to $5 \mathrm{mg} / \mathrm{kg}$ kaempferol and D-GalN/LPS in mice (Fig. 3c). These data suggest that kaempferol pretreatment maybe facilitates autophagosomes and inhibits autophagy flux in ALF mice induced by D-GalN/LPS.

\section{Effects of kaempferol on starvation-induced autophagy in vitro}

To further substantiate our results of the experiment in vitro, in starvation conditions, we evaluated the effects of kaempferol on primary hepatocytes. To observe the formation of autophagosomes, the GFPLC3 plasmid was transfected into hepatocytes. Our data shown in Fig. 4a, compared to that of the control group, the GFP-LC3 signal was weak in the starvation group and high-dose kaempferol groups but was bright and punctate in the low-dose kaempferol groups. The protein expression levels of LC3, p62 and Atg7 were measured by western blot (Fig. 4b). The starvation and low-dose kaempferol groups showed the higher protein expression levels of LC3, and the high-dose kaempferol group showed lower expression levels compared with the control group. Furthermore, the low-dose kaempferol groups showed reduced protein expression level of p62. 
The presence of autophagic flux was demonstrated by increased expression of LC3 and decreased expression of p62. Thus, our results suggest that pretreatment with low doses of kaempferol promotes induction of autophagic flux and that pretreatment with high doses of kaempferol restrains induction of autophagic flux in starvation-induced hepatocytes in vitro.

\section{Kaempferol ameliorates injury in the livers of ALF mice through autophagy mechanisms.}

Our previous studies confirmed that $5 \mathrm{mg} / \mathrm{kg}$ kaempferol had a significant effect on ALF induced by DGaIN/LPS. To further confirm whether pretreatment with $5 \mathrm{mg} / \mathrm{kg}$ kaempferol contributes to the induction of autophagy to protect against liver injury. Atg7 knockdown by siRNA was used in vivo. Our results revealed that 3-MA or Atg7 siRNA partially negated kaempferol-mediated hepatoprotection in ALF mice, in agreement with the levels of sALT and SAST in Fig. 5a, and the histology that relatively less wellpreserved liver architecture in Fig. 5b. Furthermore, we also measured the expression of LC3II and p62 when 3-MA pretreatment. The resulted showed that compared with mice treated with kaempferol ( $5 \mathrm{mg} / \mathrm{kg}$ ) and D-GalN/LPS, the 3-MA pretreatment further decreased conversion of LC3II and further increased the degradation of p62 (Fig. 3c). These dates further indicated that pretreatment with $5 \mathrm{mg} / \mathrm{kg}$ kaempferol ameliorated liver injury by regulating autophagy in ALF mice.

\section{Discussion}

Kaempferol is an ingredient in traditional Chinese herbs and is found in various vegetables and fruits, including tomatoes, citrus, grapefruit, onion, broccoli, cabbage and apples. Kaempferol, a flavonoid, has been shown to possess a broad spectrum of pharmacological activities, such as antidiabetic, antioxidative, cardioprotective, angiogenic and anticancer activities [24,25]. A previous study revealed that kaempferol can suppress liver gluconeogenesis by restraining the activity of pyruvate carboxylase and glucose- 6 phosphatase, increase hepatic glucose metabolism and insulin resistance in obese mice induced by diet [26]. In our study, we revealed that pretreatment with kaempferol at different doses had different functional effects on D-GalN/LPS-induced ALF. Our results showed that $5 \mathrm{mg} / \mathrm{kg}$ of kaempferol pretreatment significantly protected against liver injury induced by D-GaIN/LPS in mice, while kaempferol at a high dose decreased the survival rate and induced more severe injury. As a result, when using kaempferol to treat ALF, it is important to pay attention to the different effects caused by different doses and choose the appropriate dose for treatment.

ALF have a high mortality rate with clinical symptoms including coagulopathy, hepatic dysfunction and abnormal liver biochemical values. In addition, ALF is closely related to the inflammatory response and is an injury process associated with inflammation-mediated hepatocellular carcinoma. There is currently no effective treatment for ALF. Our previous research proved that endoplasmic reticulum stress can reduce inflammation by regulating the immune mechanism in ALF [27]. This study showed that $10 \mathrm{mg} / \mathrm{kg}$ and $20 \mathrm{mg} / \mathrm{kg}$ of kaempferol pretreatment increased expression of proinflammatory cytokines, and the expression of proinflammatory cytokines were remarkably decreased in pretreatment with $2.5 \mathrm{mg} / \mathrm{kg}$ and $5 \mathrm{mg} / \mathrm{kg}$ of kaempferol in ALF induced by D-GaIN/LPS. Therefore, these data suggested that high-dose 
kaempferol pretreatment can promote the hepatic inflammatory response, and low-dose kaempferol pretreatment can significantly suppress the hepatic inflammatory response in ALF mice induced by DGalN/LPS.

Autophagy is a self-digestive process that can maintain cell homeostasis, supply a variety of substrates for cellular energy generation and ensure cell survival under stressful conditions. Autophagy have a significance for the processes of cell death regulation in specific tissues, such as the liver and brain [28]. Autophagy and inflammation are closely related [29]. Our previous research found that PPARa activation alleviates the inflammatory response by promoting autophagy in ALF mice induced by D-GaIN/LPS [30]. Moreover, our data confirmed that inhibition of GSK3 $\beta$ activity increased PPARa expression and decreased the inflammatory response by further increasing autophagy [27]. Our study demonstrated that different doses of kaempferol had differential influences on the induction of autophagy and autophagosome formation in vivo and in vitro. The presence of autophagic flux demonstrated by increasing LC3 expression combined with decreased p62 expression [31]. Our date indicated that lowdose of kaempferol upregulated genes related with autophagy, increased LC3II conversion and p62 degradation; while high-dose kaempferol decreased LC3II conversion and p62 degradation, and increased autophagosome formation; the pretreatment with CQ did not significantly change the effect of $5 \mathrm{mg} / \mathrm{kg}$ of kaempferol on the expression of LC3 and p62 in ALF mice induced by D-GaIN/LPS. We therefore concluded that inflammatory response was alleviated by pretreating kaempferol with a low dose to protect mice from ALF induced by D-GaIN/LPS via autophagy pathway. However, we also believe that the effect of kaempferol on the autophagic flux at different concentrations is a very complicated physiological phenomenon and deserves further to be explored in ALF.

\section{Conclusions}

In this study, our study suggested that effects of kaempferol on ALF at different doses have different functional by regulating the autophagy pathway, and kaempferol at a low dose can significantly protect mice from liver injury in ALF. Therefore, we should select the optimal dose when kaempferol is clinically used for the effective strategy to treat ALF. For further preclinical studies of autophagy agonists, it is necessary to develop clinically applicable therapeutic strategies for ALF.

\section{Abbreviations}

ALF, acute liver failure; ALI, acute liver injury; D-GaIN, D-galactosamine; LPS, lipopolysaccharide; NAFLD, nonalcoholic fatty liver disease; 3-MA, 3-Methyladenine

$\mathrm{CQ}$, chloroquine; $\mathrm{ALT}$, alanine aminotransferase; $\mathrm{AST}$, aspartate aminotransferase; $\mathrm{H} \& \mathrm{E}$, hematoxylin and eosin; qRT-PCR, quantitative reverse-transcription polymerase chain reaction; RIPA, radioimmunoprecipitation assay; TBST, Tris-buffered saline with Tween-20; sALT, serum ALT; sAST, serum AST; IL, interleukin; CXCL, C-X-C motif chemokine ligand. 


\section{Declarations}

Acknowledgements

Not applicable.

\section{Authors' contributions}

Y.T. and F.R. wrote original draft and performed an experiment; L.X. and H.W. collected the data and performed formal analysis; W.L. and M.L. performed statistical analyses and data curation; J.H. prepared the samples and supervision; F.R. and X.Z. provided funding acquisition and designed the experiments. All authors contributed to the manuscript, which submission is approved.

\section{Funding}

This study was supported by the National Natural Science Foundation of China (81770611), the Demonstrating Application and Research of Clinical Diagnosis and Treatment Technology in Beijing (Z191100006619096 and Z191100006619097), Key Projects of the Beijing Municipal Education Commission's Science and Technology Plan (KZ202010025035), Beijing Municipal Administration of Hospitals Clinical Medicine Development of Special Funding Support (XMLX201830), Key Medical Major of Beijing Sailing Plan (ZYLX201819), the National Science and Technology Key Project on "Major Infectious Diseases such as HIV/AIDS, Viral Hepatitis Prevention and Treatment" (2018ZX10301407-005002 and 2018ZX10302205-004-004), Beijing Talents foundation (2018000021469G289), Scientific Research Project of Beijing Youan Hospital, Capital Medical University (YNKTQN20180202) and National Natural Science Foundation of China (82002243)

\section{Availability of data and materials}

The data used to support the findings of this study are available from the corresponding author upon reasonable request.

\section{Ethics approval and consent to participate}

All animal experiments were reviewed and approved by Institutional Animal Care and Use Committee (IACUC) of Capital Medical University (CMU).

\section{Consent for publication}

Not applicable.

\section{Competing interest}

The authors declare that there are no conflicts of interest.

\section{Author details}


${ }^{1}$ Beijing Youan Hospital, Capital Medical University, No. 8, XitouTiao Road, Youwai Street, Fengtai District, Beijing 100069, China.

\section{References}

1. Wendon J, Members P, Cordoba J, Dhawan A, Larsen FS, Manns M, et al. EASL Clinical Practical Guidelines on the management of acute (fulminant) liver failure. Journal of Hepatology. 2017; 66: 1047.

2. Rajendran P, Rengarajan T, Nandakumar N, Palaniswami R, Nishigaki Y, Nishigaki I. Kaempferol, a potential cytostatic and cure for inflammatory disorders. European journal of medicinal chemistry. 2014; 86: 103-112.

3. Sharifi-Rad M, Fokou PVT, Sharopov F, Martorell M, Ademiluyi AO, Rajkovic J, et al. Antiulcer Agents: From Plant Extracts to Phytochemicals in Healing Promotion. Molecules (Basel, Switzerland). 2018; 23: 1751.

4. Imran M, Salehi B, Sharifi-Rad J, Aslam Gondal T, Saeed F, Imran A, et al. Kaempferol: A Key Emphasis to Its Anticancer Potential. Molecules. 2019; 24: 2277.

5. Mahobiya A, Singh TU, Rungsung S, Kumar T, Chandrasekaran G, Parida S, et al. Kaempferol-induces vasorelaxation via endothelium-independent pathways in rat isolated pulmonary artery. Pharmacological reports: PR. 2018; 70: 863-874.

6. Imran M, Salehi B, Sharifi-Rad J, Aslam Gondal T, Saeed F, Imran A, et al. Kaempferol: A Key Emphasis to Its Anticancer Potential. Molecules (Basel, Switzerland). 2019; 24: 2277.

7. Torres-Villarreal D, Camacho A, Castro H, Ortiz-Lopez R, de la Garza AL. Anti-obesity effects of kaempferol by inhibiting adipogenesis and increasing lipolysis in 3T3-L1 cells. Journal of physiology and biochemistry. 2019; 75: 83-88.

8. Kashafi E, Moradzadeh M, Mohamadkhani A, Erfanian S. Kaempferol increases apoptosis in human cervical cancer HeLa cells via PI3K/AKT and telomerase pathways. Biomedicine \& Pharmacotherapy. 2017; 89: 573-577.

9. Wu P, Meng X, Zheng H, Zeng Q, Chen T, Wang W, et al. Kaempferol Attenuates ROS-Induced Hemolysis and the Molecular Mechanism of Its Induction of Apoptosis on Bladder Cancer. Molecules. 2018; 23: 2592.

10. Murakami T, Kohno K, Ninomiya K, Matsuda H, Yoshikawa M. Medicinal foodstuffs. XXV. Hepatoprotective principle and structures of ionone glucoside, phenethyl glycoside, and flavonol oligoglycosides from young seedpods of garden peas, Pisum sativum L. Chem Pharm Bull (Tokyo). 2001; 49: 1003-1008.

11. Xiong Q, Fan W, Tezuka Y, Adnyana IK, Stampoulis P, Hattori M, et al. Hepatoprotective effect of Apocynum venetum and its active constituents. Planta Med. 2000; 66: 127-133.

12. Matsuda $H$, Ninomiya $K$, Shimoda $H$, Yoshikawa M. Hepatoprotective principles from the flowers of Tilia argentea (linden): structure requirements of tiliroside and mechanisms of action. Bioorg Med 
Chem. 2002; 10: 707-712.

13. García-Mediavilla V, Crespo I, Collado PS, Esteller A, Sánchez-Campos S, Tuñón MJ, et al. The antiinflammatory flavones quercetin and kaempferol cause inhibition of inducible nitric oxide synthase, cyclooxygenase-2 and reactive C-protein, and down-regulation of the nuclear factor kappaB pathway in Chang Liver cells. Eur J Pharmacol. 2007; 557: 221-229.

14. Tsai M, Wang Y, Lai Y, Tsou H, Liou G, Ko J, et al. Kaempferol protects against propacetamol-induced acute liver injury through CYP2E1 inactivation, UGT1A1 activation, and attenuation of oxidative stress, inflammation and apoptosis in mice. Toxicology Letters. 2018; 290: 97-109.

15. Wang H, Chen L, Zhang X, Xu L, Xie B, Shi H, et al. Kaempferol protects mice from d-GaIN/LPSinduced acute liver failure by regulating the ER stress-Grp78-CHOP signaling pathway. Biomedicine \& Pharmacotherapy. 2019; 111: 468-475.

16. McEwan DG. Host-pathogen interactions and subversion of autophagy. Essays In Biochemistry. 2017; 61: 687.

17. Jiao L, Zhang H, Li D, Yang K, Tang J, Li X, et al. Regulation of glycolytic metabolism by autophagy in liver cancer involves selective autophagic degradation of HK2 (hexokinase 2). Autophagy. 2018; 14: 671-684.

18. Madrigal-Matute J, Cuervo AM. Regulation of Liver Metabolism by Autophagy. Gastroenterology. 2016; 150: 328-339.

19. Ren F, Zhang L, Zhang X, Shi H, Wen T, Bai L, et al. Inhibition of glycogen synthase kinase $3 \beta$ promotes autophagy to protect mice from acute liver failure mediated by peroxisome proliferatoractivated receptor $a$. Cell Death \&Amp; Disease. 2016; 7: e2151.

20. Mignon A, ROUQUET N, Fabre M, MARTIN S, Pagès J, DHAINAUT JF, et al. LPS Challenge in Dgalactosamine-Sensitized Mice Accounts for Caspase-dependent Fulminant Hepatitis, not for Septic Shock. American journal of respiratory and critical care medicine. 1999; 159: 1308-1315.

21. Nakama T, Hirono S, Moriuchi A, Hasuike S, Nagata K, Hori T, et al. Etoposide prevents apoptosis in mouse liver with D-galactosamine/lipopolysaccharide-induced fulminant hepatic failure resulting in reduction of lethality. Hepatology. 2001; 33: 1441-1450.

22. Klaunig JE, Goldblatt PJ, Hinton DE, Lipsky MM, Chacko J, Trump BF. Mouse liver cell culture. I. Hepatocyte isolation. In vitro. 1981; 17: 913-925.

23. Zhang X, Ding J, Gou C, Wen T, Li L, Wang X, et al. Qingchangligan formula attenuates the inflammatory response to protect the liver from acute failure induced by $\mathrm{d}$ galactosamine/lipopolysaccharide in mice. J Ethnopharmacol. 2017; 201: 108-116.

24. Devi KSP, Mishra D, Roy B, Ghosh SK, Maiti TK. Assessing the immunomodulatory role of heteroglycan in a tumor spheroid and macrophage co-culture model system. Carbohydrate Polymers. 2015; 127: 1-10.

25. Zang Y, Zhang L, Igarashi K, Yu C. The anti-obesity and anti-diabetic effects of kaempferol glycosides from unripe soybean leaves in high-fat-diet mice. Food Funct. 2015; 6: 834-841. 
26. Alkhalidy H, Moore W, Wang A, Luo J, McMillan RP, Wang Y, et al. Kaempferol ameliorates hyperglycemia through suppressing hepatic gluconeogenesis and enhancing hepatic insulin sensitivity in diet-induced obese mice. The Journal of Nutritional Biochemistry. 2018; 58: 90-101.

27. Ren F, Zhou L, Zhang X, Wen T, Shi H, Xie B, et al. Endoplasmic reticulum stress-activated glycogen synthase kinase $3 \beta$ aggravates liver inflammation and hepatotoxicity in mice with acute liver failure. Inflammation. 2015; 38: 1151-1165.

28. Russo M, Russo GL. Autophagy inducers in cancer. Biochem. Pharmacol. 2018; 153: 51-61.

29. Fésüs L, Demény MÁ, Petrovski G. Autophagy Shapes Inflammation. Antioxidants \& Redox Signaling. 2010; 14: 2233-2243.

30. Jiao M, Ren F, Zhou L, Zhang X, Zhang L, Wen T, et al. Peroxisome proliferator-activated receptor a activation attenuates the inflammatory response to protect the liver from acute failure by promoting the autophagy pathway. Cell Death Dis. 2014; 5: e1397.

31. Klionsky DJ, Abdelmohsen K, Abe A, Abedin MJ, Abeliovich H, Acevedo Arozena A, et al. Guidelines for the use and interpretation of assays for monitoring autophagy (3rd edition). Autophagy. 2016; 12: $1-222$.

\section{Figures}

a

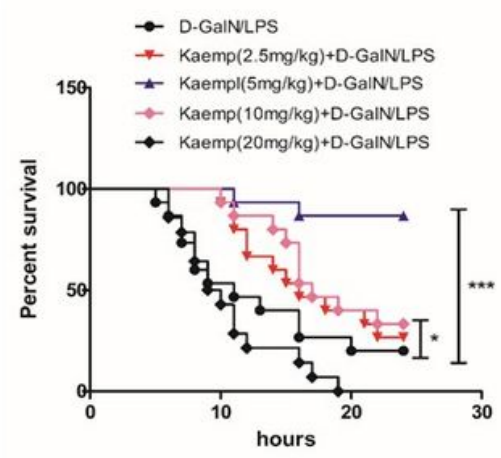

$\mathrm{c}$

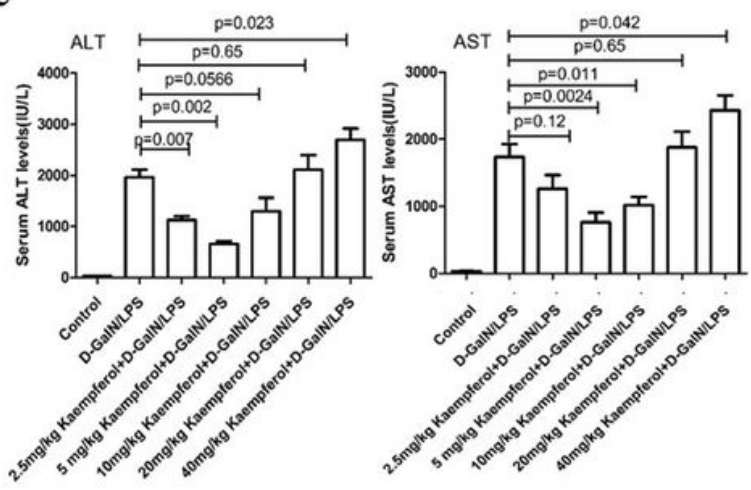

b
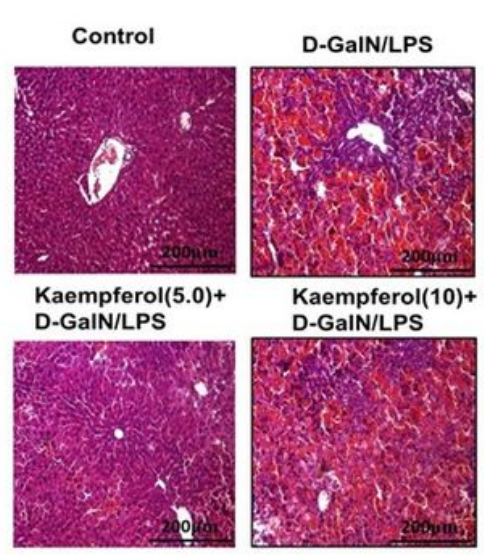

Kaempferol(2.5)+

D-GalN/LPS

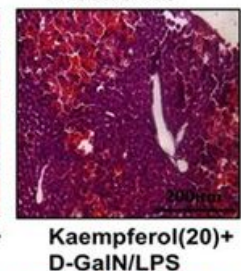

D-GalN/LPS

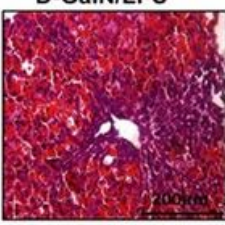

Figure 1

d
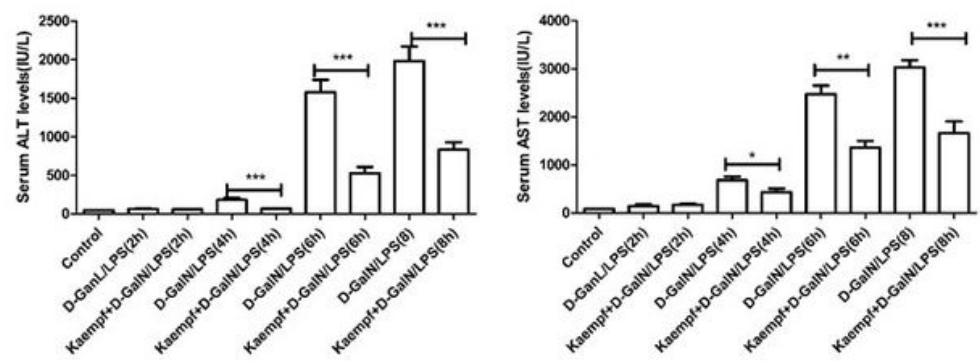

\section{Figure 1}


Effects of different doses of kaempferol on D-GalN/LPS-induced ALF. Mice treated with kaempferol + DGalN/LPS that were administered kaempferol $(2.5 \mathrm{mg} / \mathrm{kg}, 5 \mathrm{mg} / \mathrm{kg}, 10 \mathrm{mg} / \mathrm{kg}, 20 \mathrm{mg} / \mathrm{kg}$ and $40 \mathrm{mg} / \mathrm{kg}$, iv) prior to or after D-GaIN/LPS injection $(n=20)$. Control mice were pretreated with saline before $D$ GalN/LPS injection $(n=20)$. A. Survival rates of mice in the kaempferol with different doses $+D-$ GaIN/LPS-treated group and the PBS + D-GaIN/LPS-treated group (20 mice per group). *: $p<0.05, * \star *$ : $<$ 0.001 , compare with the D-GalN/LPS group, every concentration was analyzed. B. Serum AST and ALT enzyme levels in the different groups. C. H\&E-stained sections of kaempferol with different doses + DGaIN/LPS-treated mice compared with D-GaIN/LPS-treated mice. D. Serum AST and ALT enzyme levels in the different groups, including kaempferol $(5 \mathrm{mg} / \mathrm{kg})$ pretreatment, to evaluate liver injury in mice at $2 \mathrm{~h}, 4$ $h, 6 \mathrm{~h}$ and $8 \mathrm{~h}$ after D-GalN/LPS administration. $*$ : $p<0.05, * *: p<0.01, * \star *: p<0.001$.

Figure 2

a
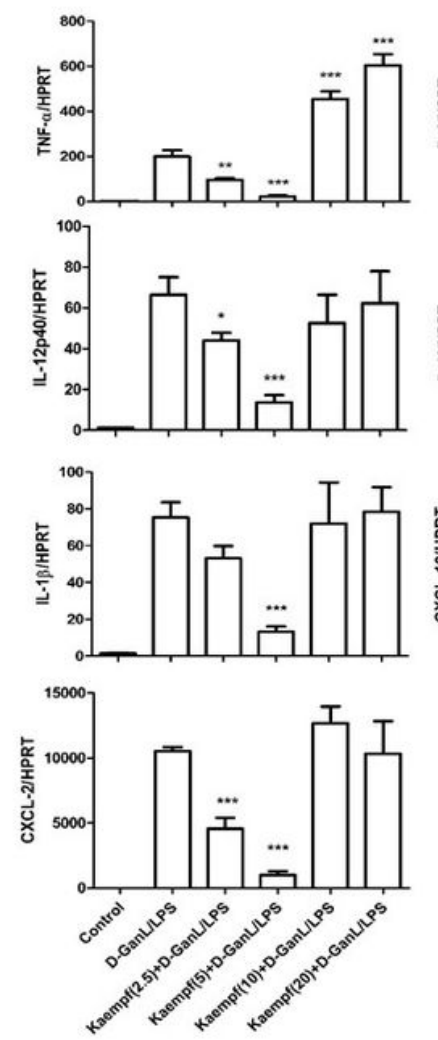

$\mathrm{b}$

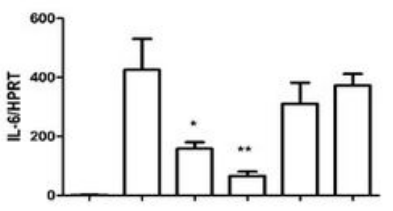

b
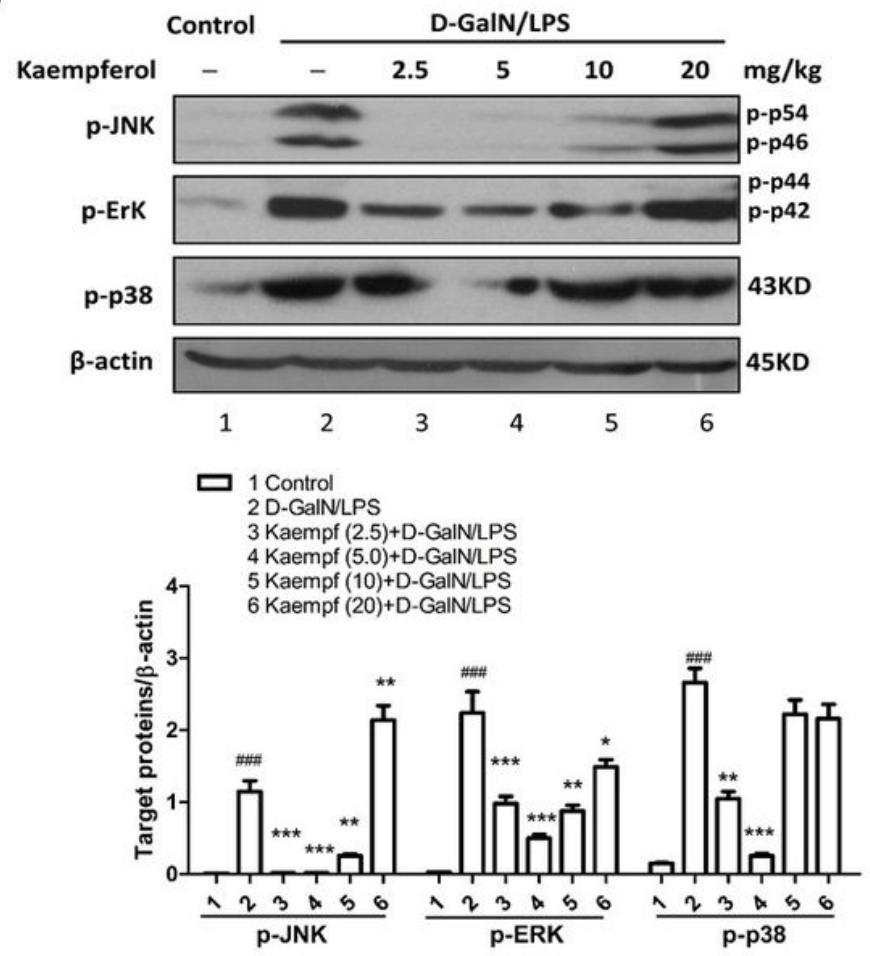

\section{Figure 2}

Effects of different doses of kaempferol on liver inflammation in D-GalN/LPS- induced ALF. A. qRT-PCR analysis was performed to determine gene expression levels of cytokines and chemokines, including TNF-a, IL-6, IL-12p40, IL-10, IL-1 , CXCL-10 and CXCL-2, in livers from mice in the different groups. *: $p<$ $0.05, * *: p<0.01, \star \star \star: p<0.001$, compare with the D-GalN/LPS group, every concentration was analyzed. $B$. Western blotting analysis was performed to determine hepatic protein expression levels of $p$-JNK, $p$ ERK, $p$-p38 and $\beta$-actin (mean \pm SD). \#\#\#: $p<0.001$, compared with control group; $*$ : $p<0.05, * *$ : $p<0.01$, $\star \star *: p<0.001$, compared with D-GalN/LPS group. 
a

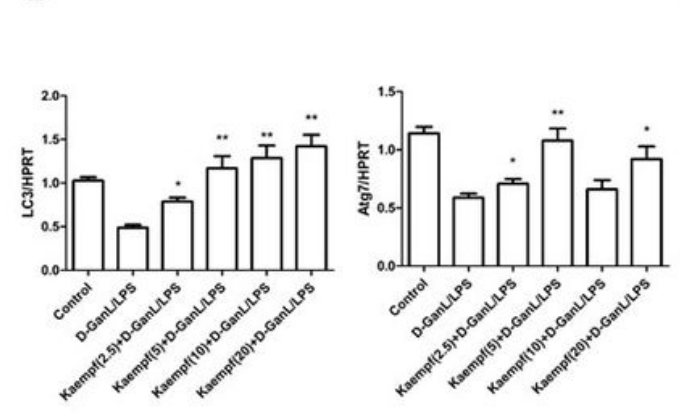

b

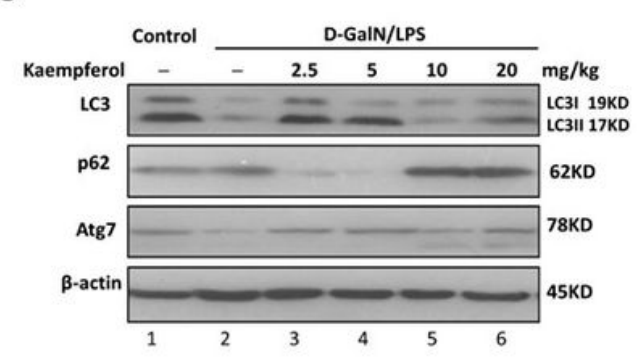

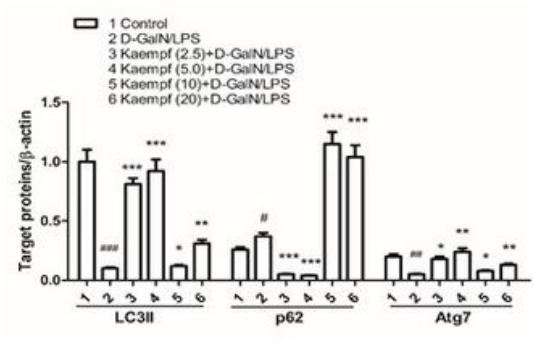

c
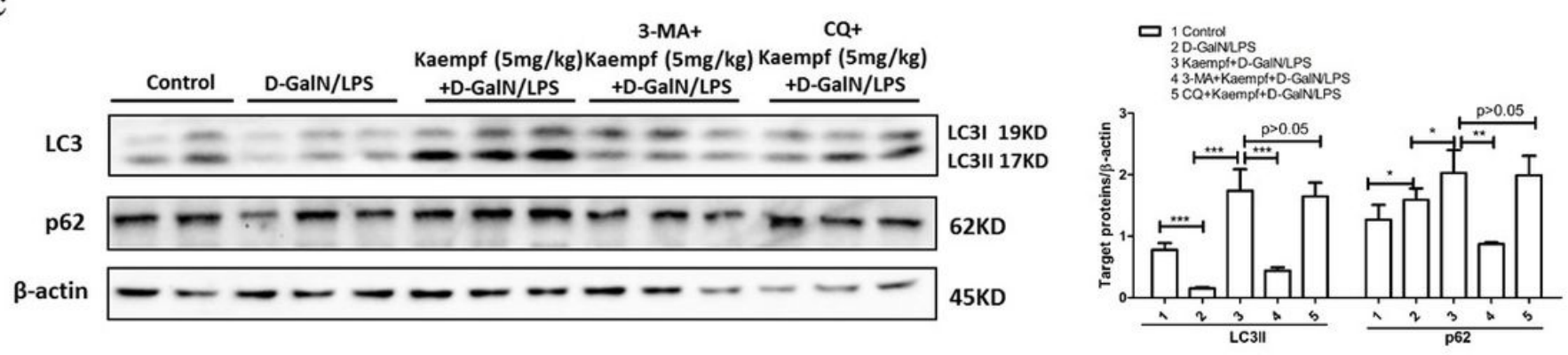

\section{Figure 3}

Effects of different doses of kaempferol on liver autophagy in D-GaIN/LPS-induced ALF. A. Gene expression levels of LC3, Atg7 and HPRT in livers from mice in the different groups were measured by qRT-PCR. *: $p<0.05$, **: $p<0.01$, compared with D-GalN/LPS group. B. Western blotting analysis was performed to determine hepatic protein expression levels of LC3, p62, Atg7 and $\beta$-actin (mean \pm SD). \#: $p$ $<0.05$, \#\#: $p<0.01$, \#\#\#: $p<0.001$, compared with control group; *: $p<0.05, * *: p<0.01$, ***: $p<0.001$, compared with D-GaIN/LPS group. C. Mice were pretreated for 2 hours with or without kaempferol (5 $\mathrm{mg} / \mathrm{kg}$, i.p.), and then stimulated with $\mathrm{D}-\mathrm{GaIN} / \mathrm{LPS}$ for 6 hours $(\mathrm{n}=12)$; mice treated with 3-MA + kaempferol + D-GaIN/LPS that were co-administered 3-MA (10 mg/kg) and kaempferol at $2 \mathrm{~h}$ before DGaIN/LPS injection ( $n=12)$; mice treated with CQ + kaempferol + D-GaIN/LPS that were co-administered CQ $(60 \mathrm{mg} / \mathrm{kg})$ and kaempferol at $2 \mathrm{~h}$ before D-GaIN/LPS injection $(\mathrm{n}=12)$; After $2 \mathrm{~h}$ vehicle injection, control mice were pretreated with PBS $(n=12)$. Western blotting analysis was performed to determine hepatic protein expression level of LC3, p62 and $\beta$-actin (mean \pm SD). *: $p<0.05, * *$ : $p<0.01, * * *$ : $p<0.001$. 
a

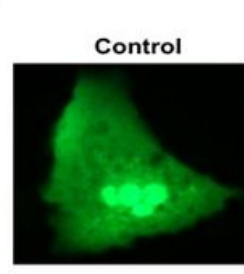

Kaempferol(0.1 $\mu \mathrm{M})$ + Starvation

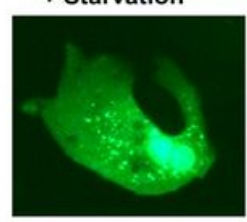

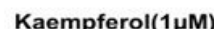
+ Starvation

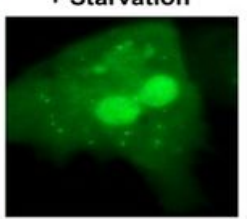

Kaempferol(10uM) + Starvation
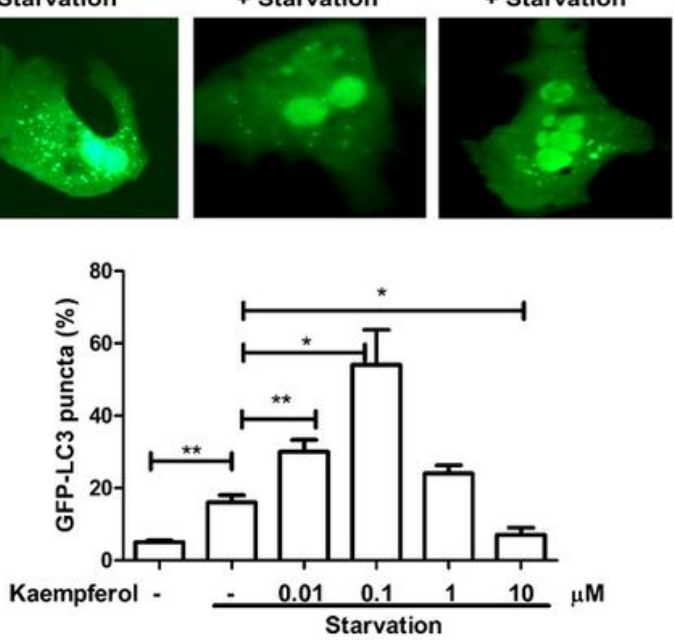

b

Figure 4

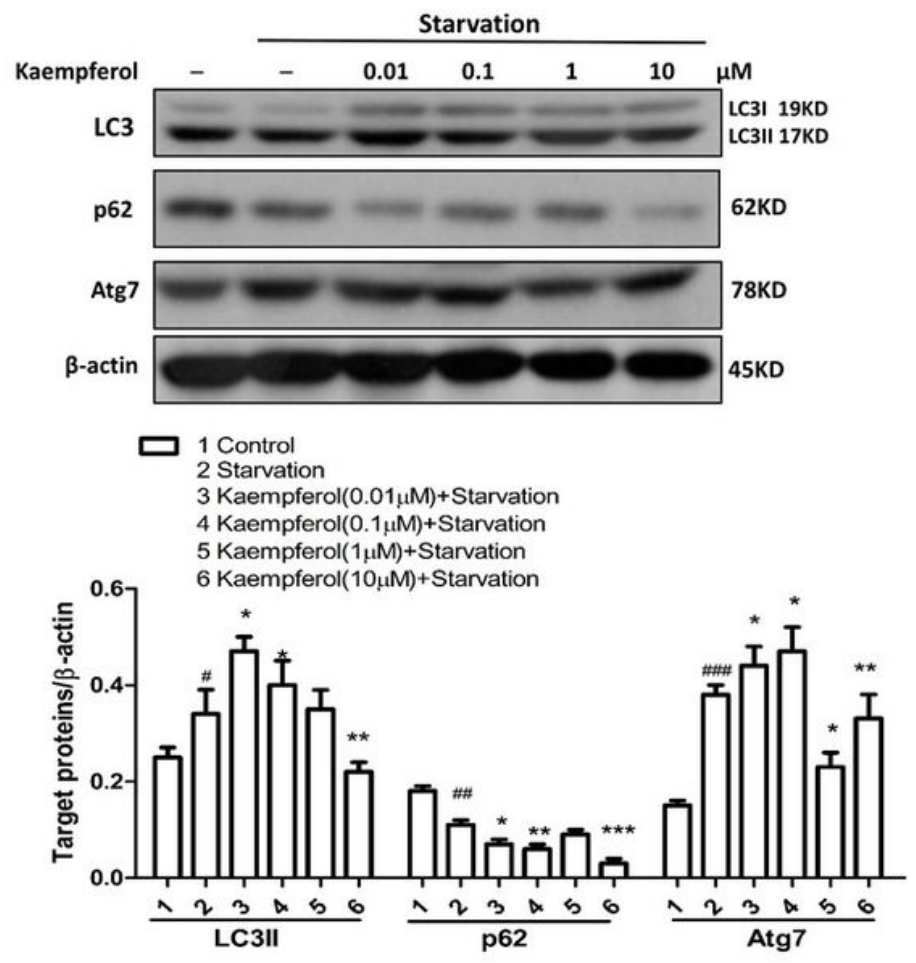

\section{Figure 4}

Effects of different doses of kaempferol on starvation-induced autophagy in vitro. A. Primary hepatocytes were transfected GFP-LC3 plasmids for $12 \mathrm{~h}$ and preincubated with kaempferol $(0.01,0.1,1$ or $10 \mu \mathrm{M})$ for $12 \mathrm{~h}$, and the formation of autophagosomes was observed. $\#$ : $\mathrm{p}<0.05$, compared with control group; *: $\mathrm{p}$ $<0.05, * *$ : $p<0.01 \rrbracket$ compared with starvation group. B. Western blotting analysis was performed to determine protein expression levels of autophagy-related proteins, including LC3, p62, Atg7, in primary hepatocytes under starvation conditions (mean \pm SD). \#: $p<0.05$, \#\#: $p<0.01$, \#\#\#: $p<0.001$, compared with control group; *: $p<0.05, * *$ : $p<0.01, * \star *$ : $p<0.001$, compared with starvation group. 
a

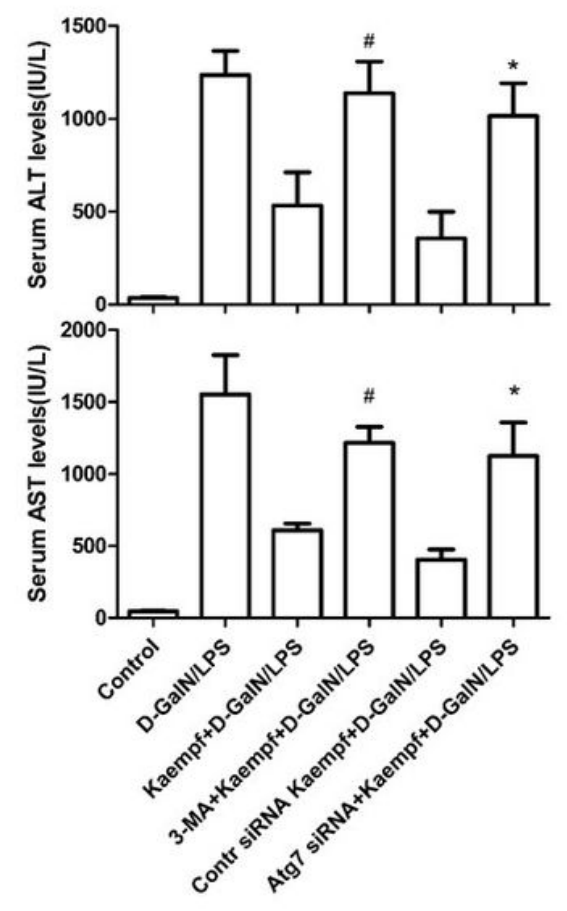

$\mathrm{b}$

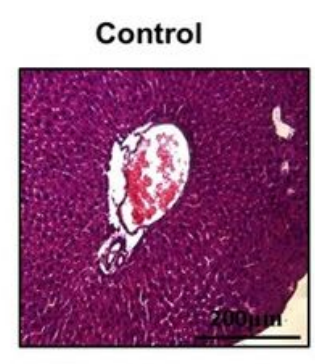

D-GaIN/LPS
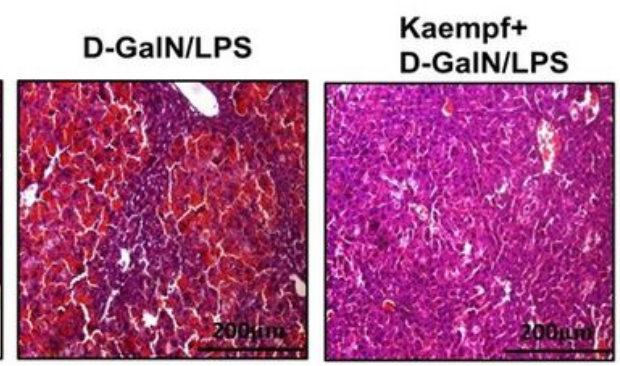

3-MA+Kaempf+

D-GaIN/LPS

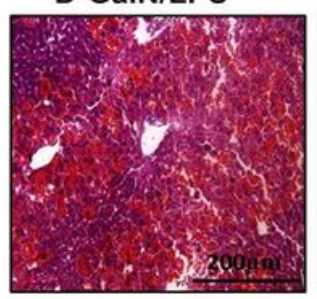

Contr siRNA+Kaempf Atg7 siRNA+Kaempf

+ D-GalN/LPS

+ D-GaIN/LPS

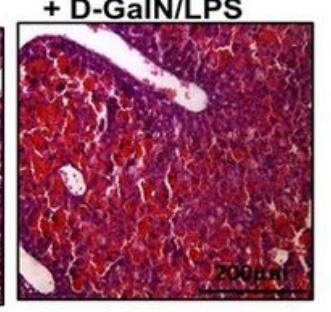

\section{Figure 5}

Kaempferol protects mice from ALF by regulating the autophagy pathway in the liver. Mice were pretreated for 2 hours with or without kaempferol ( $5 \mathrm{mg} / \mathrm{kg}$, i.p.), and then stimulated with D-GalN/LPS for 6 hours $(n=20)$; mice treated 3-MA + kaempferol + D-GalN/LPS that were administered 3-MA (10 $\mathrm{mg} / \mathrm{kg}$ ) and kaempferol $2 \mathrm{~h}$ before D-GalN/LPS injection ( $\mathrm{n}=20)$; mice treated with control siRNA or siAtg7 + kaempferol + D-GalN/LPS that were injected to tail vein for $48 \mathrm{~h}$ with control siRNA or Atg7 siRNA ( $3 \mathrm{mg} / \mathrm{kg})$ and were administered kaempferol $2 \mathrm{~h}$ before D-GaIN/LPS injection $(\mathrm{n}=20)$. After $2 \mathrm{~h}$ vehicle injection, control mice were pretreated with PBS $(n=20)$. A. Serum AST and ALT enzyme levels in the different groups. \#: $p<0.05$, compared with kaempferol + D-GalN/LPS group; *: $p<0.05$, compared with kaempferol + D-GalN/LPS + control siRNA group. B. Representative H\&E-stained of liver sections from different groups are shown. 